\title{
A clinical observation of stage I implant breast reconstruction for mass-like granulomatous lobular mastitis
}

\author{
Chaojie Zhang ${ }^{1 \#}$, Yaqin Wu ${ }^{2 \#} \wedge$, Huiling Wang ${ }^{3}$, Jie Zeng ${ }^{1}$, Shanshan Lei ${ }^{1}$, Jie He ${ }^{1}$, Zheng Zeng ${ }^{3}$, \\ Runzhang $\mathrm{Wu}^{2}$, Qian $\mathrm{Li}^{2}$, Peizhi Fan ${ }^{1}$ \\ ${ }^{1}$ Department of Breast and Thyroid Surgery, Hunan Provincial People's Hospital/The First Affiliated Hospital of Hunan Normal University, \\ Changsha, China; ${ }^{2}$ Department of Breast and Thyroid Surgery, The First Affiliated Hospital of Hunan Normal University/Hunan Provincial \\ People's Hospital, Changsha, China; ${ }^{3}$ The Second Department of Breast and Thyroid Surgery, Hunan Provincial People's Hospital/The First \\ Affiliated Hospital of Hunan Normal University, Changsha, China \\ Contributions: (I) Conception and design: C Zhang, Y Wu; (II) Administrative support: H Wang; (III) Provision of study materials or patients: J Zeng, \\ J He, S Lei, Z Zeng; (IV) Collection and assembly of data: C Zhang, H Wang, Y Wu, R Wu, Q Li; (V) Data analysis and interpretation: C Zhang, Y \\ Wu, P Fan, H Wang; (VI) Manuscript writing: All authors; (VII) Final approval of manuscript: All authors. \\ "These authors contributed equally to this work and should be considered co-first authors. \\ Correspondence to: Huiling Wang. The Second Department of Breast and Thyroid Surgery, Hunan Provincial Peoples Hospital/The First Affiliated \\ Hospital of Hunan Normal University, Changsha 410005, China. Email: 27528957@qq.com.
}

Background: Granulomatous lobular mastitis (GLM) is a chronic benign inflammatory breast disease, and mainly mass-like granulomatous lobular mastitis (MGLM) clinically. There are few reports on applications of stage I implant breast reconstruction in GLM. This observational study was conducted to evaluate the safety and efficacy of stage I implant breast reconstruction in the treatment of MGLM.

Methods: Patients suffering from MGLM who visited at hospital from April 2019 to June 2020 were selected and graded according to the magnetic resonance imaging (MRI) examination. Patients with MGLM were grouped into the prosthesis implantation group and the traditional treatment group according to their preferences. Clinical parameters of the two groups were analyzed before and after surgery, such as postoperative infection, recurrence, and satisfaction with appearance and aesthetics were observed. To evaluate the safety and efficacy of the implant breast reconstruction in MGLM.

Results: There were 59 cases of MGLM, 31 cases of grade 3-4 GLM, 11 cases of bilateral metachronous GLM. There were 18 patients in the prosthesis implantation group, including 9 patients with bilateral metachronous GLM, 2 patients with synchronous GLM, and 41 patients in the traditional treatment group. All the patients were followed up with a median of 17.5 months. One patient in the observation group had an infection on the reconstructed side 3 weeks after surgery, and the implant was retained after 2 weeks of conservative treatment such as antibiotics. Two patients in the prosthesis implantation group were not satisfied with size of the reconstructed breast was smaller than the opposite side. In the traditional treatment group, there were 3 cases of postoperative infection or delayed wound healing, and 26 cases of postoperative breast asymmetry.

Conclusions: For patients with MGLM, it is safe to select stage I prosthesis implantation after conservative treatment, with exact effect and high patient satisfaction.

Keywords: Granulomatous lobular mastitis (GLM); mass-like; stage I; implant breast reconstruction

Submitted Jun 24, 2021. Accepted for publication Aug 02, 2021.

doi: $10.21037 /$ gs-21-417

View this article at: https://dx.doi.org/10.21037/gs-21-417

$\wedge$ ORCID: 0000-0002-9275-607X. 


\section{Introduction}

Granulomatous lobular mastitis (GLM), also known as granulomatous mastitis, idiopathic granulomatous mastitis (IGM), was divided into mass-like granulomatous lobular mastitis (MGLM) and non-mass type GLM (1) according to the morphology of the lesion after enhanced magnetic resonance imaging (MRI). GLM is a chronic non-infectious inflammatory disease that can cause breast deformation. It was first described as a benign inflammatory disease of the breast by Kessler and Wolloch in 1972 (2). GLM was relatively rare in clinical practice in the past. However, the incidence of GLM has increased sharply in recent years (3), mainly in Asia and Mediterranean countries, such as China, Iran and Turkey $(4,5)$. The etiology and pathogenesis of GLM are still unclear. Many hypotheses of which have been born at present, such as autoimmune disorders (which was widely supported), infection (especially infection caused by Corynebacterium), pregnancy, lactation, hyperprolactinemia, and so on, all of which may be related to the pathogenesis of GLM (6-9). Nowadays, Corynebacterium infection continues to be a hotspot of the current research in the etiology of GLM. However, the relevant studies of our team have found no absolute correlation between the incidence of GLM and Corynebacterium (10). One of the most recognized possible causes is the autoimmune inflammatory response caused by exudate from lobules (11). Our study also confirmed that GLM may be an inflammatory disease related to autoimmunity (12).

GLM usually occurs in women, and it also occurs in a small number of men (13). GLM mainly manifests as painful lumps with signs of inflammation in the breast, nipple discharge, nipple retraction, persistent abscess, sinuses, and fistula $(8,14,15)$, the former two is up to $50 \%$ of GLM patients. Some patients are complicated with erythema nodosum or arthritis. Fifteen to fifty-five percent of GLM patients have ipsilateral reactive axillary lymph node enlargement (11). GLM is often unilateral breast (11), yet may involve both breasts. However, no explanation has been proven to explain the timing of pathogenesis of bilateral cases. At present, there are no standard treatment guidelines for GLM, and main treatment is surgical. Meanwhile, inappropriate timing and method of surgery may lead to distorted breast appearance. Furthermore, the recurrence rate of traditional surgery is as high as $50 \%(6)$, and the continuous course of the disease and repeated breast surgery has a huge impact on women's physical and mental health $(16,17)$.
MGLM is more common in childbearing age women who are also more keen on breast appearance. For a large range of lesions, high rate of postoperative recurrence, the protracted illness will lead to physical and mental pressure. This study evaluated the safety and efficacy of mastectomy with stage I implant breast reconstruction in the treatment of MGLM by comparing the clinical data, grade, onset time, complications, and satisfaction of MGLM patients with different treatment modalities in a small sample study. We present the following article in accordance with the STROBE reporting checklist (available at https://dx.doi. org/10.21037/gs-21-417).

\section{Methods}

\section{Clinical materials}

Patients suffering from MGLM who visited at hospital from April 2019 to June 2020 were selected and graded according to the MRI examination. Patients underwent core needle aspiration biopsy and MRI. For MGLM, we proposed to divide it into four grades, namely, grade 1 involving 1 site, grade 2 involving 2 sites, grade 3 involving 3 sites, and grade 4 involving 4 or more sites (the nidus encroaches a quadrant or the rear of nipple areola as a site). The research flowchart is shown in Figure 1.

\section{Inclusion criteria}

This retrospective study was conducted in accordance with the Declaration of Helsinki (as revised in 2013). The study was approved by the Hamilton Integrated Research Ethics Board (approval \#2017SK2142) and the HoffmannLa Roche global review committee. All patients enrolled completed the informed consent form and met the diagnostic criteria for MGLM.

\section{Exclusion criteria}

Patients with autoimmune diseases, other serious systemic diseases, serious heart, liver and renal insufficiency, coagulation dysfunction, contraindications to surgery, pregnant women, and patients who refuse surgery were excluded from the study.

\section{Treatment}

Prosthesis implantation group (observation group)

Preoperative conservative treatment, including traditional 


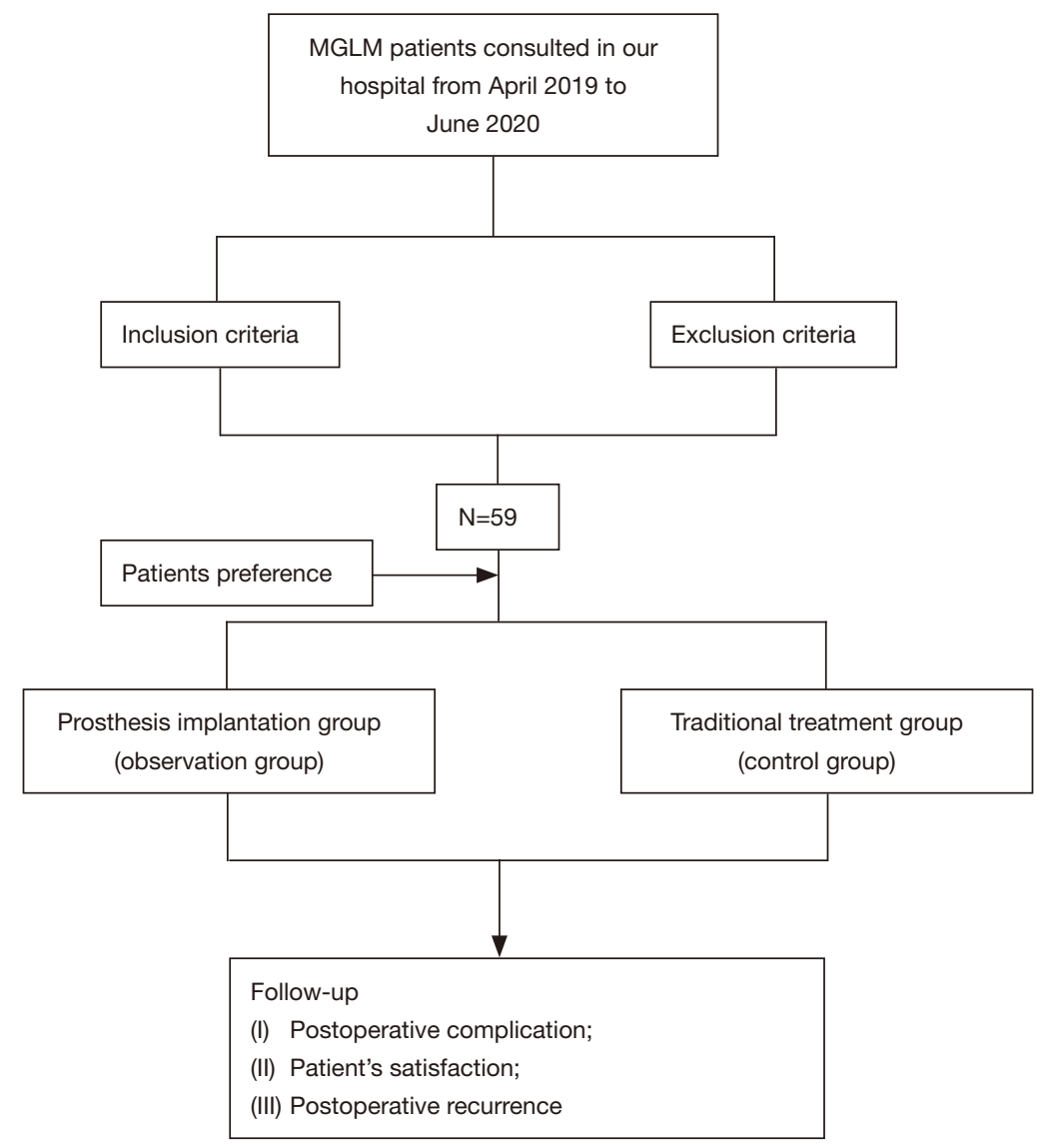

Figure 1 Research flowchart. MGLM, mass-like granulomatous lobular mastitis.

Chinese medicine, antibiotics, the local wet packing, abscess drainage, and so on, were used to control acute inflammation. The patient underwent stage I silicone prosthesis implantation after subcutaneous skin-sparing mastectomy. After preoperative examination and relevant preparations, routine preoperative skin disinfectant and draping were performed. After anesthesia, an incision was made at the anterior axillary line, and a subcutaneous skin-sparing mastectomy + retaining pectoralis major fascia with stage I prosthesis implantation was performed. Postoperative routine indwelling drainage, antibiotics antiinfection treatment.

\section{Traditional treatment group (control group)}

Preoperative conservative treatment, including traditional Chinese medicine, antibiotics, the local wet packing, abscess drainage, and so on, were used to control acute inflammation. The patient underwent surgery for removal of the lesion. After preoperative examination and relevant preparations, routine preoperative skin disinfectant and draping were performed. After anesthesia, an incision was made at the anterior axillary line to remove all lesions until normal tissues were obtained. Postoperative routine indwelling drainage, antibiotics anti-infection treatment.

\section{Efficacy evaluation}

We have analyzed preoperative and postoperative clinical features of two groups of patients and observed the rate of postoperative infection, fat liquefaction, and recurrence during the follow-up period. According to clinical observation and summary of our research team, there was no recurrence followed up for 1 year was defined as cure. The safety and efficacy of the prosthesis implantation group were estimated by subjective evaluation of patient's satisfaction: very satisfied (patients feel satisfied), satisfied (patients think it is still acceptable or better than no treatment), and dissatisfied (feeling dissatisfied and 
Table 1 The comparison with grade and time of onset between the observation group and the control group

\begin{tabular}{lcc}
\hline Group & $\begin{array}{c}\text { Prosthesis } \\
\text { implantation group }\end{array}$ & $\begin{array}{c}\text { Traditional treatment } \\
\text { group }\end{array}$ \\
\hline MGLM grade 1-2 & 5 & 23 \\
MGLM grade 3-4 & 13 & 18 \\
Synchronous GLM & 2 & 0 \\
Metachronous GLM & 9 & 0
\end{tabular}

MGLM, mass-like granulomatous lobular mastitis; GLM, granulomatous lobular mastitis.

Table 2 The complication of the observation group and the control group

\begin{tabular}{lcc}
\hline \multirow{2}{*}{ Group } & \multicolumn{2}{c}{ Complication } \\
\cline { 2 - 3 } & Infection & Fat liquefaction \\
\hline Prosthesis implantation group & 1 & 0 \\
Traditional treatment group & 1 & 2 \\
\hline
\end{tabular}

regretting the choice of this method).

\section{The postoperative follow-up}

After the end of treatment, regular reexaminations were conducted at 3, 6, 9 and 12 months after surgery. All patients were followed up by telephone and outpatient service, and the number of recurrent cases in each group was recorded.

\section{Statistical analysis}

Statistical analyses were carried out by SPSS 26.0 (IBM Corporation, Armonk, NY, USA) statistical package. Measurement data and enumeration data were represented as mean \pm standard deviation and number or rate (\%), respectively. $W$ test was used for the normal distribution test, and $\mathrm{F}$ test was used for homogeneity of variance test. The $t$-test was used for comparison between groups. The cross-table $\chi^{2}$ test was used for a rate comparison. $\mathrm{P}<0.05$ was considered statistically significant.

\section{Results}

\section{Clinical data}

A total of 59 MGLM patients presented at our hospital from April 2019 to June 2020 and were selected. MGLM was confirmed in patients by core needle aspiration biopsy and MRI. According to MRI examination and evaluation, the patients were divided into grade 1-4 MGLM, and divided into the prosthesis implantation group (observation group) and the traditional treatment group (control group) according to their preference. The patients ranged in age from 25 to 56 years, with a median age of 34.5 years. The follow-up time was from 10 to 25 months, and the median follow-up time was 17.5 months. There was no significant difference in age and other general data between the two groups $(\mathrm{P}>0.05)$. A total of 28 patients with MGLM grade $1-2,5$ of whom $(17.9 \%)$ chose prosthesis implantation and $23(82.1 \%)$ chose traditional treatment; 31 patients with grade $3-4,13$ of whom $(41.9 \%)$ chose prosthesis implantation and 18 (58.1\%) chose traditional treatment (Table 1).

\section{Efficacy and follow-up results}

No patient was lost to follow-up. No recurrence was found in the observation group $\left(\mathrm{n}_{1}=18\right)$ and the control group $\left(\mathrm{n}_{2}=41\right)$ during the follow-up period. There were 11 cases of contralateral breast lesions during the follow-up period, which were pathologically confirmed as GLM, including 2 cases of synchronous GLM and 9 cases of metachronous GLM (Table 1). Postoperative infection occurred in 1 patient in each of the two groups, the observation group $(5.6 \%)$ and the control group $(2.4 \%)$. One patient in the observation group had an infection on the prosthesis side 3 weeks after surgery, and the prosthesis was retained after 2 weeks of conservative treatment such as antibiotics. In the control group, 2 patients occurred fat liquefaction (4.9\%), that is, postoperative complications occurred in 3 patients $(7.3 \%)$. No patients in the observation group occurred fat liquefaction after surgery (Table 2). In the observation group, 2 patients $(11.1 \%)$ were dissatisfied, 6 patients $(33.3 \%)$ were satisfied, and 10 patients (55.6\%) were very satisfied. In the control group, 26 patients $(63.4 \%)$ were dissatisfied, 10 patients $(24.4 \%)$ were satisfied, and 5 patients $(12.2 \%)$ were very satisfied (Table 3). There are two cases in different groups, case 1 in observation group (Figure 2) and case 2 in control group (Figure 3).

\section{Discussion}

GLM is a chronic inflammatory benign breast disease that often occurs in women during the child-bearing 
Table 3 The comparison with appearance satisfaction between the observation group and the control group

\begin{tabular}{lcccc}
\hline \multirow{2}{*}{ Group } & \multicolumn{3}{c}{ Appearance satisfaction } & \multirow{2}{*}{$\chi^{2}$} \\
\cline { 2 - 4 } & Dissatisfied & Satisfied & Very satisfied & 16.830 \\
Prosthesis implantation group & 2 & 6 & 10 & $<0.05^{*}$ \\
Traditional treatment group & 26 & 10 & 5 & \\
\hline
\end{tabular}

${ }^{*}$, statistically significant difference $(\mathrm{P}<0.05)$.
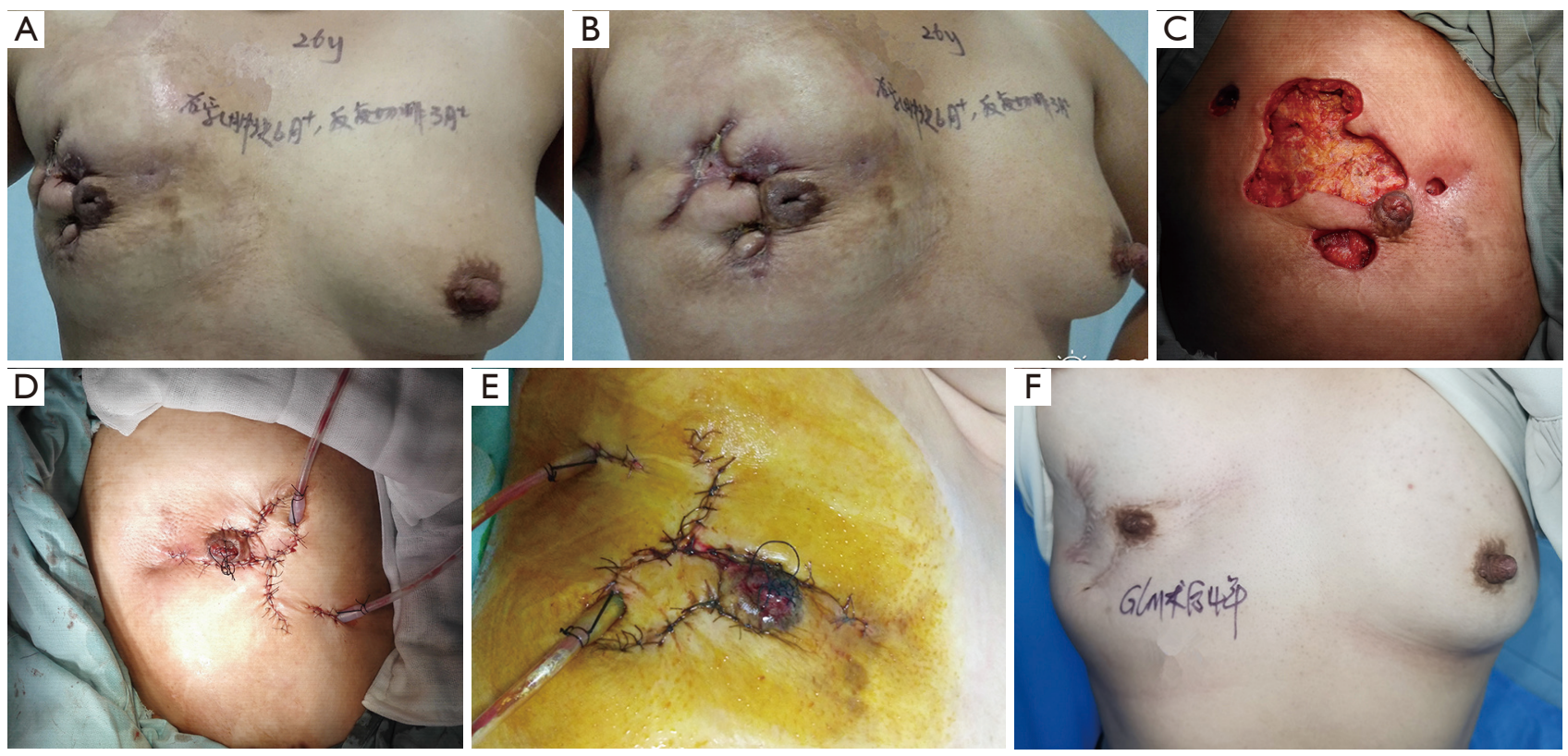

Figure 2 Case 1: a 26-year-old female with "right breast swelling and pain 6 month". (A,B) Physical examination: congenital severe nipple retraction of the right breast, local skin scar formation, and stretching to breast deformation. There were no palpated significantly enlarged lymph nodes in the bilateral axilla, no erythema nodosum was observed throughout the body; (C,D) the right breast lesions were removed; (E) 3 days after removal of the lesion; (F) 4 years after removal of the lesion.

period. With an average age of 30 (18). The disease usually develops rapidly, and mainly MGLM clinically. We have consulted literature at home and abroad, and have not found in literature an explanation for the synchronous and metachronous of bilateral GLM. Based on our clinical experience and the time limit definition of bilateral breast cancer (19), synchronous breast cancer was defined as bilateral breast onset $\leq 12$ months, and metachronous breast cancer was defined as bilateral breast onset $>12$ months. We consider that if the contralateral breast develops GLM within 1 year, then it can be considered as synchronous GLM, and if the ipsilateral breast develops GLM within 1 year, then it can be considered as a recurrence. The course of GLM can be as long as 1 to 2 years (20). There are no specific imaging manifestations of GLM, and color doppler ultrasound is the most commonly used method. The most common manifestations of color doppler ultrasound of the breast: multiple continuous hypoechoic masses or irregular inhomogeneous echoic masses with the enhanced posterior acoustic shadow or posterior echo, blurred edges, and effusion and cavity in the later stage, combined with cutaneous fistula $(11,15)$. Mammogram: unilateral or locally asymmetric, with increased density, ill-defined boundaries, or irregular masses (single or multiple), skin thickening, and axillary lymph node enlargement, and 24\% GLM patients had no abnormal findings on the mammogram $(11,21)$. Our study group believes that mammography is not recommended for patients with breast inflammatory diseases, as mammography requires extrusion of the breast, which is easy to cause damage to the galactophore 

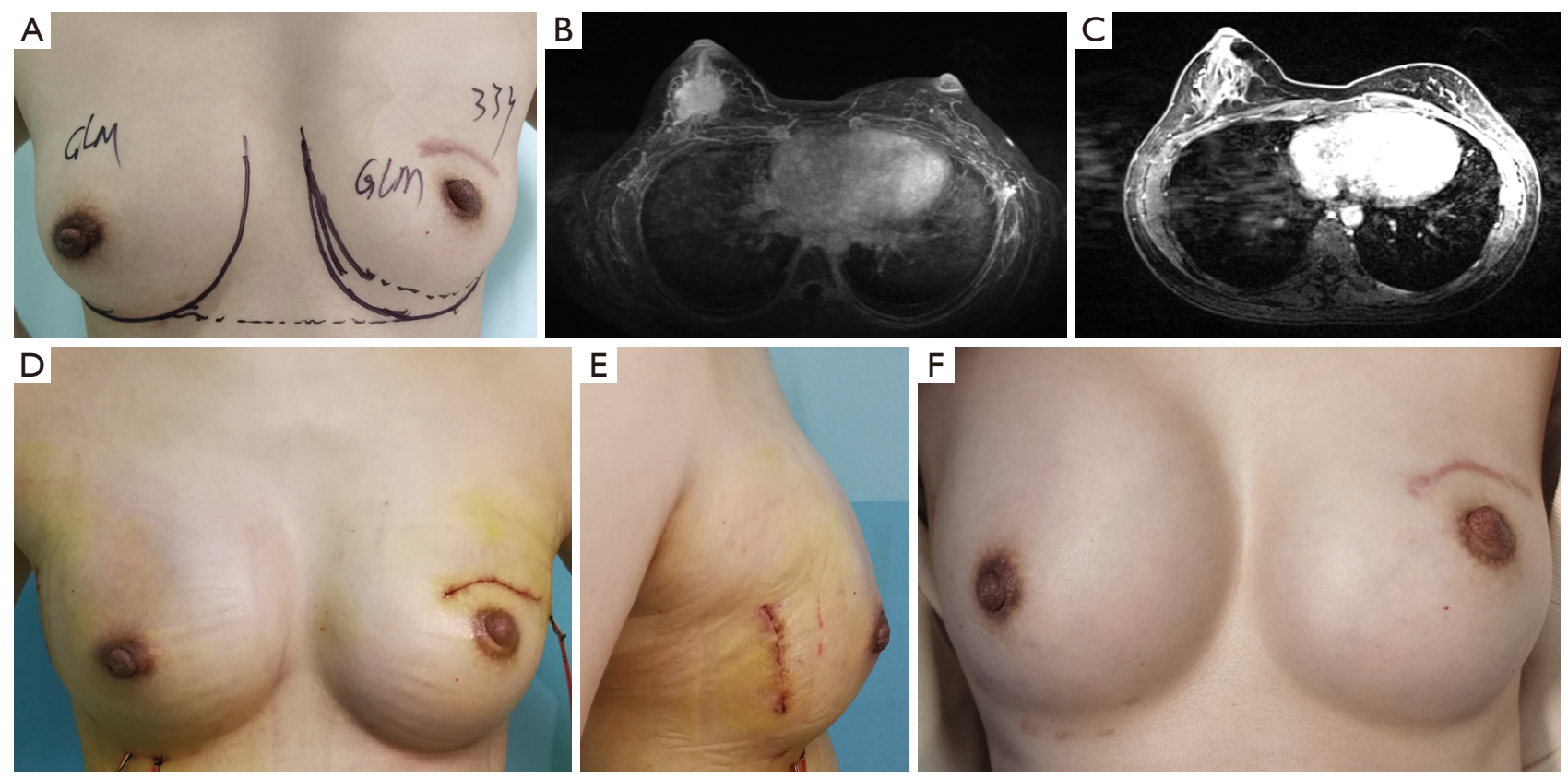

Figure 3 Case 2: a 33-year-old female with "right breast mass accompanied by swelling and pain for more than half a month". (A) Physical examination: there was an old surgical scar with a length of $4.5 \mathrm{~cm}$ in the upper quadrant of the left breast without palpable solitary nodules; there was a hard mass with a size of about $6 \mathrm{~cm} \times 5 \mathrm{~cm}$ in the upper quadrant of the right breast accompanied by tenderness; there was no local skin redness and swelling; the skin temperature was not high; there was no palpable significantly enlarged lymph nodes in the bilateral axilla; no erythema nodosum was observed throughout the body; (B,C) enhanced MRI: a mass-like enhancement in the center of the right breast, BI-RADS 4a (granulomatous mastitis?), left breast BI-RADS 2; (D,E) right subcutaneous skin-sparing mastectomy + retaining pectoralis major fascia with stage I silicone prosthesis implantation + left breast prosthesis implantation under general anesthesia; (F) 3 months after the operation. MRI, magnetic resonance imaging; BI-RADS, Breast Imaging Reporting and Data System.

and may aggravate the spread of inflammatory diseases. MRI results are variable, and uneven and ambiguous mass enhancement is more common (11). Since the clinical manifestations and auxiliary examinations of GLM are non-specific, the initial clinician is prone to misdiagnose GLM as other diseases. GLM should be differentiated from other easily misdiagnosed diseases at first, and the most important thing is to differentiate it from inflammatory breast cancer (22). GLM can only be confirmed by histopathology, and core needle aspiration biopsy is the gold standard with a sensitivity of $96 \%$ (11). All patients in this study undergo core needle aspiration biopsy. The pathologic features are nonnecrotic granulomas with local infiltrations of multinucleated giant cells, epithelioid histiocytes, lymphocytes, and plasma cells. The formation of centrilobular granulomas and microabscesses can be observed (Figure 4).

The etiology of GLM is still unknown and there are no clear guidelines or expert consensus for the best first-line treatment. Optional treatments include clinical observation, oral antibiotics, steroid therapy, antituberculous therapy, traditional Chinese medicine, and surgical treatment current (15). GLM is aseptic inflammation by definition, so antibiotics are usually ineffective (23), and there is no effective treatment for Corynebacterium at present. Some researchers have recommended high-dose steroid shock therapy (24), but there are many side effects of corticosteroid therapy, such as weight gain, hyperglycemia, and Cushing syndrome. A small number of patients who failed corticosteroid treatment received methotrexate (25), but there are many adverse reactions of methotrexate. Therefore, whether methotrexate could be used in women of reproductive age should be considered (26). Topical steroid steroids may also be used in patients who refuse oral hormone therapy and who have mild symptoms.

The high frequency of disease recurrence is one of the principal reasons affecting the physical and mental health of patients with GLM. Some studies (27) have confirmed 

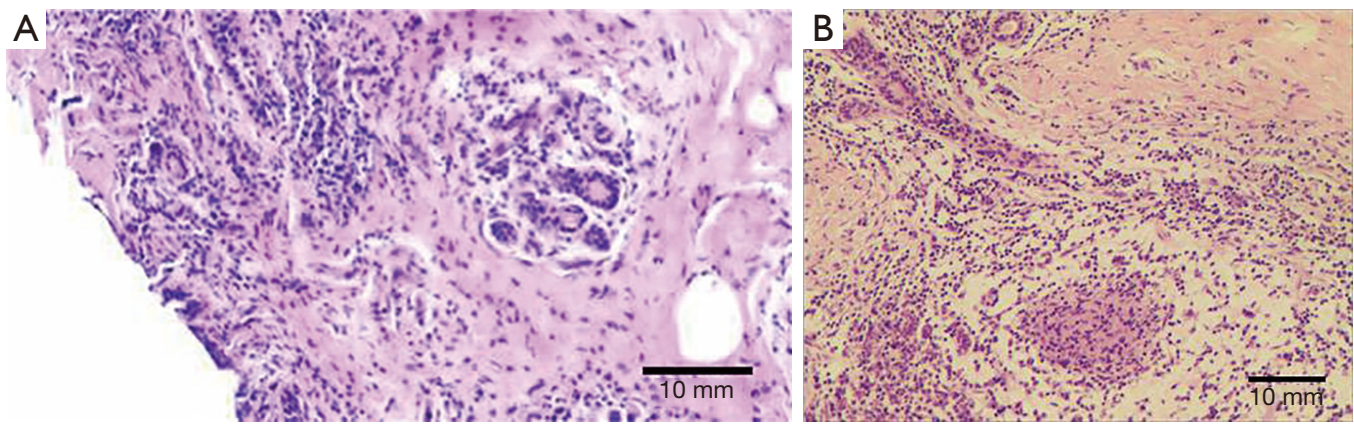

Figure 4 Histopathological results by hematoxylin-eosin staining in patients with GLM. (A) Nonnecrotic granuloma with more lymphocytes and plasma cells; (B) centrilobular granuloma and abscess formation were observed. GLM, granulomatous lobular mastitis.

that there are different recurrence risks for different treatment methods. Antibiotics having the lowest efficacy, with an improvement rate of $6-21 \%$, and corticosteroid treatment with a success rate of $66-72 \%$. Lei et al. (28) have reported recurrence rates of $20 \%$ with oral steroids, $6.8 \%$ with surgery alone, or $4 \%$ with surgery combined steroids. A meta-analysis (29) showed that surgery significantly achieved a higher rate of complete response (CR) and a lower rate of recurrence than hormone therapy, as well as a shorter duration of treatment. Therefore, surgery should be the primary method of treatment for GLM. The timing of surgery depends on the personalized clinical evaluation of the patient, so does the choice of method of operation, which includes incision and drainage of the breast abscess, simple lumpectomy, breast lesion removal, and subcutaneous skin-sparing mastectomy, as well as breast reconstruction according to the patient's will (30). In this study, postoperative complications and recurrence of patients in the prosthesis implantation group (observation group) and the traditional treatment group (control group) were followed up. No recurrence was found in patients in the two groups during the follow-up period. Postoperative infection occurred in 1 patient in each group, and the control group had fat liquefaction after surgery, that is to say, 3 cases of postoperative complications in the control group (7.3\%). No postoperative fat liquefaction was observed in patients in the observation group. The incidence of postoperative complications in the observation group is lower than that in the control group, but the difference has no significance. The follow-up time is short, and patients who choose stage I prosthesis implantation may have capsular contracture and other complications in the future, so further follow-up and observation are needed. Meanwhile, we need to expand the sample size for further study to induce more guiding significance results for clinical treatment, as our included data of small sample study are less.

The first approach of surgical treatment of GLM is to remove the focus and surrounding affected tissue thoroughly. GLM has a wide range of lesions (31), especially for patients with grade 3 or 4 of MGLM, whose lesions almost invade the whole breast. The traditional operation will damage the aesthetic of the breast, influence the appearance of the breast, aggravate the psychological burden and social barriers of patients (16). It is demonstrated that the satisfaction and quality of life of patients with postoperative breast reconstruction are higher than those without breast reconstruction (20). Our research group previously confirmed that GLM patients underwent breast lesion excision and stage I breast reconstruction by transposition with intraglandular and fascia flap (LE + BR), which is a safe and effective treatment method and has a good cosmetic effect, short recovery time, and low recurrence rate (32). However, LE + BR also has its limitations, which requires that the range of lesions of MGLM patients is less than or equal to 1 quadrant, which limits the extensive clinical use of LE + BR. In this study, patients in the observation group received a subcutaneous skin-sparing mastectomy + retaining pectoralis major fascia with stage I prosthesis implantation, which can be applied to all MGLM patients, and whose application range is far greater than that of autologous tissue flap reconstruction. We cannot be only to quantitative indicators to judge due to different severity of GLM with variable treatment effect. Therefore, to pay more attention to the patient's subjective feelings, this study adopted the patient satisfaction questionnaire. Not only including the change of the breast shape after treatment but also including the patient's subjective 
satisfaction in the process of treatment. In our study, the "dissatisfied" of the observation group was significantly lower than that of the control group, and the rates of "satisfied" and "very satisfied" were also significantly higher than that of the control group. However, the sample size of this study was small, and the groups were not randomly divided but according to the wishes of patients, so selection bias may occur between the two groups. The research team will expand the sample size of the study in the future, and conduct satisfaction analysis based on extensive data.

Our study group believes that stage I breast reconstruction avoids the loss of breast, avoids the need for a second surgery, and decreases surgical costs and length of hospital stay. This study is a brave attempt based on solid theory. We concluded that the rate of postoperative recurrence and complication of patients in the prosthesis implantation group was not higher than those in the traditional treatment group. Meanwhile, the stage I silicone prosthesis implantation could achieve satisfactory breast cosmetic effect for patients. Therefore, in our opinion, a subcutaneous skin-sparing mastectomy + retaining pectoralis major fascia with stage I prosthesis implantation is effective in the treatment of MGLM, with high patient satisfaction, low recurrence rate and good application prospect in clinical practice, while being safe, feasible and easy-to-operate.

In conclusion, the general trend of GLM is long-term recurrence and patients suffer a huge psychological burden at the same time. Postoperative breast reconstruction provides a valuable option for patients' physical and mental resilience. In MGLM patients with large lesion areas, a subcutaneous skinsparing mastectomy + retaining pectoralis major fascia with stage I prosthesis implantation is recommended under the premise of strict control of indications. It is more necessary for experienced clinicians to make comprehensive choices based on patients' wishes to choose what kind of breast reconstruction method and when to implement it. However, more multi-center studies, a larger number of patients and a longer follow-up period are necessary to guide breast reconstruction treatment of GLM.

\section{Acknowledgments}

We would like to thank the patients that participated in this study.

Funding: This study was supported by Key Projects of China Hunan Provincial Science \& Technology Department (No. 2017SK2142).

\section{Footnote}

Reporting Checklist: The authors have completed the STROBE reporting checklist. Available at https://dx.doi. org/10.21037/gs-21-417

Data Sharing Statement: Available at https://dx.doi. org/10.21037/gs-21-417

Conflicts of Interest: All authors have completed the ICMJE uniform disclosure form (available at https://dx.doi. org/10.21037/gs-21-417). The authors have no conflicts of interest to declare.

Etbical Statement: The authors are accountable for all aspects of the work in ensuring that questions related to the accuracy or integrity of any part of the work are appropriately investigated and resolved. The study was conducted in accordance with the Declaration of Helsinki (as revised in 2013). The study was approved by the Hamilton Integrated Research Ethics Board (approval \#2017SK2142) and the Hoffmann-La Roche global review committee. Informed consent was taken from all the patients.

Open Access Statement: This is an Open Access article distributed in accordance with the Creative Commons Attribution-NonCommercial-NoDerivs 4.0 International License (CC BY-NC-ND 4.0), which permits the noncommercial replication and distribution of the article with the strict proviso that no changes or edits are made and the original work is properly cited (including links to both the formal publication through the relevant DOI and the license). See: https://creativecommons.org/licenses/by-nc$\mathrm{nd} / 4.0 /$.

\section{References}

1. Aslan H, Pourbagher A, Colakoglu T. Idiopathic granulomatous mastitis: magnetic resonance imaging findings with diffusion MRI. Acta Radiol 2016;57:796-801.

2. Kessler E, Wolloch Y. Granulomatous mastitis: a lesion clinically simulating carcinoma. Am J Clin Pathol 1972;58:642-6.

3. Oddó D, Domínguez F, Gómez N, et al. Granulomatous lobular mastitis associated with ductal carcinoma in situ of the breast. SAGE Open Med Case Rep 2019;7:2050313X19836583.

4. Al Manasra AR, Al-Hurani MF. Granulomatous Mastitis: 
A Rare Cause of Male Breast Lump. Case Rep Oncol 2016;9:516-9.

5. Velidedeoglu M, Kilic F, Mete B, et al. Bilateral idiopathic granulomatous mastitis. Asian J Surg 2016;39:12-20.

6. Huang $\mathrm{Y}, \mathrm{Wu} \mathrm{H}$. A retrospective analysis of recurrence risk factors for granulomatous lobular mastitis in 130 patients: more attention should be paied to prolactin level. Ann Palliat Med 2021;10:2824-31.

7. Sheybani F, Naderi HR, Gharib M, et al. Idiopathic granulomatous mastitis: Long-discussed but yet-to-beknown. Autoimmunity 2016;49:236-9.

8. Nikolaev A, Blake CN, Carlson DL. Association between Hyperprolactinemia and Granulomatous Mastitis. Breast J 2016;22:224-31.

9. Jiang L, Li X, Sun B, et al. Clinicopathological features of granulomatous lobular mastitis and mammary duct ectasia. Oncol Lett 2020;19:840-8.

10. Characterization of Microflora in Granulomatous Lobular Mastitis by 16S rDNA High-throughput Sequencing [dissertation]. Changsha: Hunan Normal University, 2020.

11. Wolfrum A, Kümmel S, Theuerkauf I, et al. Granulomatous Mastitis: A Therapeutic and Diagnostic Challenge. Breast Care (Basel) 2018;13:413-8.

12. Kong C, Zhang C, Wu Y, et al. The expression and meaning of CD68, CD163, CD57, and IgG4 in granulomatous lobular mastitis. Gland Surg 2020;9:936-49.

13. Pluguez-Turull CW, Nanyes JE, Quintero CJ, et al. Idiopathic Granulomatous Mastitis: Manifestations at Multimodality Imaging and Pitfalls. Radiographics 2018;38:330-56.

14. Martinez-Ramos D, Simon-Monterde L, Suelves-Piqueres $\mathrm{C}$, et al. Idiopathic granulomatous mastitis: A systematic review of 3060 patients. Breast J 2019;25:1245-50.

15. Barreto DS, Sedgwick EL, Nagi CS, et al. Granulomatous mastitis: etiology, imaging, pathology, treatment, and clinical findings. Breast Cancer Res Treat 2018;171:527-34.

16. Chen L, Zhang XY, Wang YW, et al. Granulomatous lobular mastitis: a clinicopathological analysis of 300 cases. Zhonghua Bing Li Xue Za Zhi 2019;48:231-6.

17. Liu PZ, Shi XG, Zuo XM, et al. A clinical study on the treatment of granulomatous lobular mastitis by the external application of the internal pus-expelling decoction and operation. Ann Palliat Med 2020;9:2631-41.

18. Johnstone KJ, Robson J, Cherian SG, et al. Cystic neutrophilic granulomatous mastitis associated with Corynebacterium including Corynebacterium kroppenstedtii. Pathology 2017;49:405-12.

19. Mao J, Hai J, Zhang C, et al. Clinical analysis and epidemical features of bilateral primary breast cancer. China Journal of Modern Medicine 2004;(19):105$107+110$.

20. Wang J, Zhang Y, Lu X, et al. Response to Letter to Editor RE: Idiopathic Granulomatous Mastitis with Skin Rupture: A Retrospective Cohort Study of 200 Patients Who Underwent Surgical and Nonsurgical Treatment. J Invest Surg 2020. [Epub ahead of print]. doi: 10.1080/08941939.2020.1780519.

21. Fazzio RT, Shah SS, Sandhu NP, et al. Idiopathic granulomatous mastitis: imaging update and review. Insights Imaging 2016;7:531-9.

22. Brennan ME, Morgan M, Heilat GB, et al. Granulomatous lobular mastitis: Clinical update and case study. Aust J Gen Pract 2020;49:44-7.

23. Bashir MU, Ramcharan A, Alothman S, et al. The enigma of granulomatous mastitis: A series. Breast Dis 2017;37:17-20.

24. Elzahaby IA, Khater A, Fathi A, et al. Etiologic revelation and outcome of the surgical management of idiopathic granulomatous mastitis; An Egyptian centre experience. Breast Dis 2016;36:115-22.

25. Calis H, Karabeyoglu SM. Follow-up of granulomatous mastitis with monitoring versus surgery. Breast Dis 2017;37:69-72.

26. Wong SCY, Poon RWS, Chen JHK, et al. Corynebacterium kroppenstedtii Is an Emerging Cause of Mastitis Especially in Patients With Psychiatric Illness on Antipsychotic Medication. Open Forum Infect Dis 2017;4:ofx096.

27. Yılmaz TU, Gürel B, Güler SA, et al. Scoring Idiopathic Granulomatous Mastitis: An Effective System for Predicting Recurrence? Eur J Breast Health 2018;14:112-6.

28. Lei X, Chen K, Zhu L, et al. Treatments for Idiopathic Granulomatous Mastitis: Systematic Review and MetaAnalysis. Breastfeed Med 2017;12:415-21.

29. Ma X, Min X, Yao C. Different Treatments for Granulomatous Lobular Mastitis: A Systematic Review and Meta-Analysis. Breast Care (Basel) 2020;15:60-6.

30. Li J. Diagnosis and Treatment of 75 Patients with Idiopathic Lobular Granulomatous Mastitis. J Invest Surg 2019;32:414-20.

31. Wang Y, Song J, Tu Y, et al. Minimally invasive 
comprehensive treatment for granulomatous lobular mastitis. BMC Surg 2020;20:34.

32. Zhang C, Lei S, Kong C, et al. Clinical study on surgical

Cite this article as: Zhang $\mathrm{C}, \mathrm{Wu} \mathrm{Y}$, Wang $\mathrm{H}$, Zeng J, Lei S, He J, Zeng Z, Wu R, Li Q, Fan P. A clinical observation of stage I implant breast reconstruction for mass-like granulomatous lobular mastitis. Gland Surg 2021;10(9):2663-2672. doi: $10.21037 / g s-21-417$ treatment of granulomatous lobular mastitis. Gland Surg 2019;8:712-22. 